\title{
Phenomenological Model for the metal-insulator transition in two dimensions
}

\author{
J.F. Weisz \\ INTEC(CONICET-UNL) Guemes 3450, 3000 Santa Fe Rep. Argentina
}

(Received on 16 September, 2009)

\begin{abstract}
The resistivity measured in two-dimensional MOSFET geometry is modeled by considering that the resistivity is a function of the temperature and the areal density of charges (electrons or holes). The logistics differential equation is proposed for the behaviour of the resistivity as a function of temperature, so that the two phases are obtained in a natural manner. At low temperatures, the Drude model behaviour is assumed for the resistivity as a function of density. Two characteristics then follow in a natural manner: The existance of a characteristic temperature for resistivity as a function of temperature, and the symmetry relationship. If the magnetic field is incorporated into the Drude model, reasonable results are obtained for the qualitative behaviour of resistivity for weak fields.
\end{abstract}

Keywords: metal-insulator transition, logistics equation

\section{INTRODUCTION}

The decade of the 90 s saw the emergence of very high mobility MOSFET devices, at temperatures of a few degrees Kelvin. A spate of experimental results followed on easily measurable quantities such as sample resistivity. This brought the surprise of a metalic as well as insulating phases, separated by a critical value of the resistivity of the order of $\frac{3 h}{e^{2}} \cdot[1-3]$.

This was a novelty for theorists, who expected only an insulating phase, due to the prediction of all states being localized in two dimensions. Thereafter followed some work trying to include interactions into the picture.[4, 5]. However, the general picture today is that microscopic models are so far unable to account for the overall behaviour, in all aspects of the problem.[6]

Since one is not ready yet to microscopically model the situation, with any degree of success, it is valid to resort to intermediate phenomenological models. It is in this spirit that the Logistics differential equation is proposed for the problem.

The merits advocated for this provisional model, the Logistics differential equation supplemented by the use of the Drude model at low temperatures, are the prediction of a characteristic temperature for resistivity behaviour as a function of temperature, and a proof of the symmetry relationship. Additionally, reasonable results are obtained for weak magnetic fields, used in connection with the Drude model.

In particular, it seemed to the author, that at this stage of development, the nonlinear aspects could only arise if this type of method is taken. It should be realized, that since this is a new form of analysis, and therefore the usual scaling method is not applied. However scaling ideas are implicit in the Logistics equation, and there is an effective reference temperature.

\section{RESISTIVITY MODEL}

If $\rho(T, n)$ is the resistivity, we take as the one of the central equations, the differential equation

$$
\frac{d \rho}{d T}=\alpha\left(\rho_{0}-\rho\right) \rho
$$

$\alpha$ is here a constant with respect to the temperature, but possible dependent on the areal density $n$.

This is known as the Logistics differential equation[7, 8], of often use in biology or economics, but very seldomly in Physics. It describes an inherently nonlinear situation, here two phases. The insulating phase occurrs here for $\rho>\rho_{0}$, such that the derivative of $\rho$ with respect to temperature $T$ is negative. The unexpected metalic phase, for which the derivative of $\rho$ with respect to temperature $T$ is positive, occurs for a resistivity lower than the critical value $\rho_{0}$. Here $n$ is the areal density of charges (electrons or holes).

The nonlinear, autonomous differential equation (1) is easily separated and integrated with respect to temperature. For that reason we must set some initial condition at some fixed temperature. That is why we take the Drude form at zero temperature $T=0$, so that

$$
\rho(T=0, n)=\frac{m}{n e^{2} \tau}
$$

Here $\tau$ is a scattering time, and $e$ the electric charge. This gives a plane resistivity inversely proportional to the areal charge. While experiments do not reach $T=0$, the low temperature data indeed suggests that $\rho$ behaves inversely with $n$.[9] For example the insulating phase is then associated with low values of areal density, and there is a critical density $n_{C}$ separating the two phases.

The result of the integration is that, with the definition $\beta=$ $\frac{\rho(0)}{\rho(T=0, n)}$,

$$
\frac{\rho}{\rho_{0}}=\frac{1}{1+(\beta-1) \exp \left(-\frac{T}{T_{0}}\right)}
$$

where

$$
T_{0}=\frac{1}{\alpha \rho_{0}}
$$

Let us take now $n=n_{C}+\Delta n$, and write out $\beta$ in more detail, when we are near the transition, that is $n_{C}>\Delta n$ in absolute value. It turns out that $\beta-1$ is $\frac{\Delta n}{n_{C}}$. Finally we will 
find that $\frac{\rho\left(T, n_{C}+\Delta n\right)}{\rho_{0}} \frac{\rho\left(T, n_{C}-\Delta n\right)}{\rho_{0}}$ is very close to 1 , and this is the statement of the symmetry relationship.[10, 11]

That the resistivity ratio is a function of $\frac{T}{T_{0}}$, where $T_{0}$ is possibly a function of $\mathrm{n}$, is also quite consistent with the usual scaling idea.

\section{MAGNETORESISTANCE}

The effect of a magnetic field in this case has been seen to be large at low temperatures, and has the effect of removing the metalic phase, transforming it into the insulating phase.[12, 13] At low temperatures only slight differences of carrier density cause large differences in the resistivity, due to the proximity of the metal-insulator transition. We take the case of a magnetic field perpendicular to the plane of the charge motion. However, positive magnetoresistance has only been found very near the transition, being negative elsewhere.

The appropriate modification, for weak fields, is in the Drude model, to have

$$
\rho(T=0, n)=\frac{m\left(1+\omega^{2} \tau^{2}\right)}{n e^{2} \tau}
$$

where $\omega=\frac{e B}{m}$ is the Cyclotron frequency due to the magnetic field B. (Compare with equation (2).) For a Silicon MOSFET the effective mass $m$ is about 0.19 of the actual electron mass.

\section{DISCUSSION}

It is thought that since the electronic correlations dominate the problem, with an $r_{S}$ of about 10, which is the ration of the electron-electron energy, divided by Fermi energy, that this is the reason for which single electron models should not work well. The microscopic scenario is far from understood, and the model is merely intended as an intermediate working scenario, until true microscopic understanding emerges.

A few facts are not understandable, within a purely interacting electron model. (1) Why the Drude model should seem at least qualitatively true at low temperatures. (2) A lower level of electron density leads to stronger correlations, but this gives us the insulating phase, rather than the metalic phase. It is then hard to understand the metalic phase as directly a consequence of correlations.

As opposed to purely interacting electrons, single electrons moving around in the presence of traps is alternative.[14, 15] At low density, such electrons cannot elude the traps, and the system behaves as an insulator. For higher electron density one is able to have some electrons eluding the traps, and has the metalic phase. However, one is still very far from being able to predict the detail from just this. Probably at this stage, the most promising line of attack is to combine disorder (i.e. traps) with the effect of interactions, as initiated by Finkelstein and Castellani. The reason then that the effect of correlations may be ultra important, is the effective very low concentration of charges, in excess or in deficit of the critical density, very near the transition. It would then be $\Delta n$, and not $n$ itself playing the role of carrier concentration, this giving the non-Fermi, Wigner type behaviour for a certain subset of carriers.

To say why a Drude model could hold at very low temperatures, we recall that in this situation one probably deals in collective modes, for which a quasiparticle image may apply. Experiments do not extrapolate to very low temperature, and one cannot say for sure what the behaviour really is.

What differentiates this work, is that it implies a strong role for the low temperature Drude model, with the theoretical prediction of a finite resistivity for $T=0$, and gives a sigmoid form for the overall function in terms of the ratio $T / T_{0}$. However it does not calculate the detail of the behaviour of $T_{0}$ as a function of $n$, which has been the subject of experimental measurments. Within usual scaling, $T_{0}$ is often proportional to $\left|\Delta n / n_{c}\right|^{z v}$.

On the other hand the usual phenomenological scaling fits are meant for an intermediate temperature range, and say nothing about zero temperature behaviour. What is also eventually wrong is that the resistivity at the critical density does eventually depend on $T$, for high enough temperatures, and is not temperature independent, except within the intermediate temperature range one usually deals with. This may probably be corrected when phonons are brought into a microscopic model.

The present phenomenological model gives resistivity curves, bounded above and below by a separatrix, which in this model is temperature independent. The symmetry relationship is a natural consequence.
[1] S. V. Kravchenko, G. V. Kravchenko, J. E. Furneaux, V. M. Pudalov and M. DIorio

Phys. Rev. B50, 8039 (1994)

[2] S. V. Kravchenko, M. P. Sarachik and D. Simonian Phys. Rev. Lett. 83, 2091 (1999)

[3] Elihu Abrahams, S. V. Kravchenko and M. P. Sarachik Rev. of Modern Physics, Vol. 73, No. 2, P. 251 (2001)

[4] C. C. Castellani, C. Di Castro and P. A. Lee Phys. Rev. B57, R9381 (1998)

[5] A. M. Finkelstein Z. Phys. B, Condensed Matter 56, P. 189 (1984)

[6] D. Popovic, A. B. Fowler and S. Washburn Phys. Rev. Lett. 79, 1543 (1997)
[7] P. F. Verhulst

Nouv. mem. de l'Academie Royale des Sci. et Belles-Lettress de Bruxells

18 1-41 (1845)

[8] Neil Gershenfeld, The Nature of Mathematical Modeling Cambridge University Press, Cambridge, ISBN 978-0521-570954

[9] Gerhard Brunthaler Metal-Insulator transition in two dimenison Institut fr Halbleiterphysik, Universitt Linz, A-4040 Linz, Austria

[10] D. Simonian, S.V. Kravschenko and M. P. Sarachik Phys. Rev. B55, R13421 (1997)

[11] S. V. Kravchenko Braz. Journal of Physics 29, No. 4, Dec. 
1999

[12] S. V. Kravchenko, M. P. Sarachik Rep. Prog. Phys. 67, 1 (2004)

[13] M. P. Sarachik, S. V. Kravchenko European Journal of Physics B40, No. 4, 397-402 (2004)
[14] B. L. Altshuler and D. L. Maslov Phys. Rev. Lett. 82, 145 (1999)

[15] T. M. Klapwijk and S. Das Sarma Solid State Comm. 110, 581 (1999) 